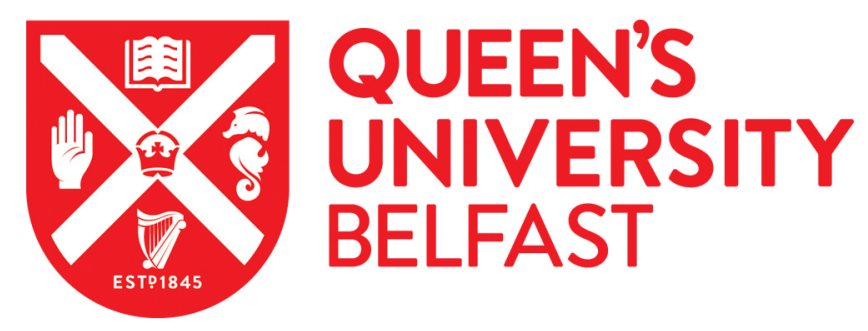

\title{
Substantial genetic divergence between morphologically indistinguishable populations of Fasciola suggests the possibility of cryptic speciation.
}

Walker, S. M., Prodöhl, P., Hoey, E. M., Fairweather, I., Hanna, R. E. B., Brennan, G., \& Trudgett, A. (2012). Substantial genetic divergence between morphologically indistinguishable populations of Fasciola suggests the possibility of cryptic speciation. International Journal for Parasitology, 42(13-14), 1193-1199. https://doi.org/10.1016/j.ijpara.2012.10.007

\section{Published in:}

International Journal for Parasitology

\section{Document Version:}

Peer reviewed version

Queen's University Belfast - Research Portal:

Link to publication record in Queen's University Belfast Research Portal

\author{
Publisher rights \\ (C) 2012 Australian Society for Parasitology Inc. Published by Elsevier Ltd. All rights reserved. \\ This manuscript is distributed under a Creative Commons Attribution-NonCommercial-NoDerivs License \\ (https://creativecommons.org/licenses/by-nc-nd/4.0/), which permits distribution and reproduction for non-commercial purposes, provided the \\ author and source are cited.
}

\section{General rights}

Copyright for the publications made accessible via the Queen's University Belfast Research Portal is retained by the author(s) and / or other copyright owners and it is a condition of accessing these publications that users recognise and abide by the legal requirements associated with these rights.

Take down policy

The Research Portal is Queen's institutional repository that provides access to Queen's research output. Every effort has been made to ensure that content in the Research Portal does not infringe any person's rights, or applicable UK laws. If you discover content in the Research Portal that you believe breaches copyright or violates any law, please contact openaccess@qub.ac.uk. 


\section{Accepted Manuscript}

Substantial genetic divergence between morphologically indistinguishable populations of Fasciola suggests the possibility of cryptic speciation

S.M. Walker, P.A. Prodöhl, E.M. Hoey, I. Fairweather, R.E.B. Hanna, G. Brennan, A. Trudgett

PII: S0020-7519(12)00252-4

DOI: http://dx.doi.org/10.1016/j.ijpara.2012.10.007

Reference: PARA 3464

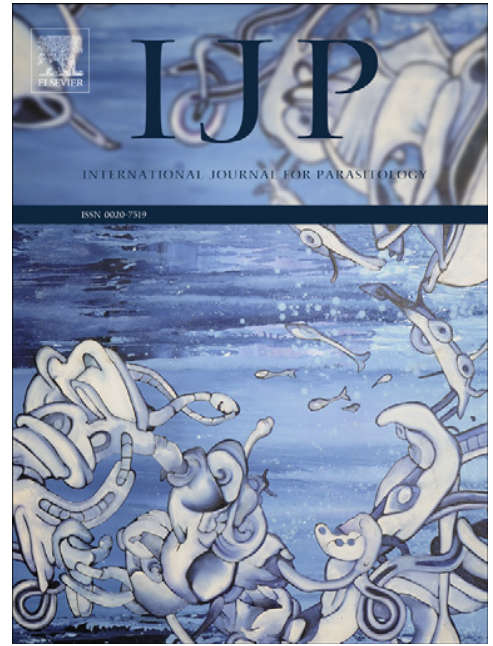

To appear in:

International Journal for Parasitology

Received Date:

13 July 2012

Revised Date:

15 October 2012

Accepted Date:

16 October 2012

Please cite this article as: Walker, S.M., Prodöhl, P.A., Hoey, E.M., Fairweather, I., Hanna, R.E.B., Brennan, G., Trudgett, A., Substantial genetic divergence between morphologically indistinguishable populations of Fasciola suggests the possibility of cryptic speciation, International Journal for Parasitology (2012), doi: http://dx.doi.org/ 10.1016/j.ijpara.2012.10.007

This is a PDF file of an unedited manuscript that has been accepted for publication. As a service to our customers we are providing this early version of the manuscript. The manuscript will undergo copyediting, typesetting, and review of the resulting proof before it is published in its final form. Please note that during the production process errors may be discovered which could affect the content, and all legal disclaimers that apply to the journal pertain. 
1 Substantial genetic divergence between morphologically indistinguishable

2 populations of Fasciola suggests the possibility of cryptic speciation $\star$

3

4 SM Walker ${ }^{\mathrm{a}}$, PA Prodöhl ${ }^{\mathrm{a}}$, EM Hoey ${ }^{\mathrm{a}}$, I Fairweather ${ }^{\mathrm{a}}$, REB Hanna ${ }^{\mathrm{b}}$, G Brennan $^{\mathrm{a}}, \mathrm{A}$

5 Trudgett ${ }^{\mathrm{a}, *}$

6

7

8

9

10

11

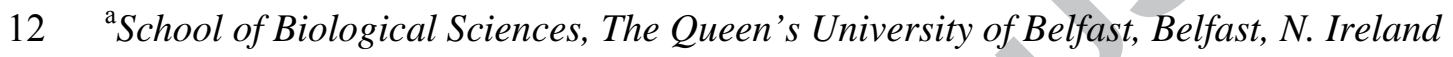
13

$14{ }^{\mathrm{b}}$ Veterinary Sciences Division, Agri-Food and Biosciences Institute, Stormont, Belfast, $15 \quad$ N. Ireland

16

17 *Corresponding author. Tel.: +44 2890792125 ; fax: +44 2890975877.

18 E-mail address: a.trudgett@qub.ac.uk

19

$20 \star$ This study contains GenBank submissions $\underline{\text { JX236561-JX236579 }}$;X236580-

$21 \quad \underline{\mathbf{J X 2 3 6 5 9 0}} ; \underline{\mathrm{JX236591-JX236639}} ; \underline{\mathrm{JX236643-JX236655}} ; \underline{\mathrm{JX236508-JX236560}}$; and

$22 \quad$ JX236640-JX236642. 


\section{Abstract}

24 The liver flukes, Fasciola hepatica and Fasciola gigantica, are considered to be sister

25 species and between them present a major threat worldwide to livestock production.

26 In this study sequence data have been employed from informative regions of the

27 nuclear and mitochondrial genomes of over 200 morphologically F. hepatica-like or

28 F. gigantica -like flukes from Europe, sub-Saharan Africa and South Asia to assess

29 genetic diversity. Evidence is presented for the existence of four well-separated

30 clades: African gigantica-like flukes, Indian gigantica-like flukes, European hepatica-

31 like flukes and African high-altitude hepatica-like flukes. Application of the

32 Biological Species Concept to trematodes is problematic; however, the degree of

33 separation between these groups was sufficient for them to be considered as distinct

34 species using the four times rule for speciation.

36 Keywords: Fasciola, Genetic diversity, Cryptic species 


\section{Introduction}

Fasciolosis, a food-borne infection by fasciolid trematodes (most commonly Fasciola hepatica or Fasciola gigantica) causes losses to agriculture estimated at US $\$ 2,000$ million per annum (McManus and Dalton, 2006). It is also an emerging zoonotic disease with up to 17 million people thought to be infected worldwide (Hopkins, 1992) and more than 90 million (Keiser and Utzinger, 2005) at risk of infection. In the developing world the economic impact of fasciolosis may be especially severe as there is a heavy reliance on buffalo and oxen as draught animals. The spread and increased incidence of fasciolosis due to climate change, drug resistance and intensification of agriculture have already been noted with $F$. hepatica in Europe and may be expected to occur with F. gigantica in the developing world (Mas Coma et al., 2009).

The evolution of $F$. hepatica and F. gigantica, together with other members of the Fasciolidae, has recently been studied in detail (Lotfy et al., 2008) and the analysis presented by these authors resulted in F. hepatica and F. gigantica being grouped as sister species. Fasciola hepatica has a cosmopolitan distribution, being reported from Europe, the Americas, Australasia and the more temperate regions of Africa and Asia, whereas F. gigantica appears to be more restricted in its range, being reported from the tropical regions of Asia and Africa, and Hawaii (introduced in the $19^{\text {th }}$ century) (Torgerson and Claxton, 1999). It is possible that outside Eurasia, the distribution of F. hepatica is the result of anthropogenic introductions in historical times to the Americas and Oceania (Mas Coma et al., 2009). Irrespective of the region, the successful establishment of either F. hepatica or F. gigantica is dependent on the distribution of its preferred intermediate host (Walker et al., 2008). Although domestic bovine and ovine herbivores sustain the bulk of the fasciolid population, 
63

64

65

66

67

both F. hepatica and F. gigantica appear to be generalist parasites with regard to their mammalian hosts and, thus, they are capable of infecting a wide range of species (Torgerson and Claxton, 1999). This lack of specialisation with regard to the definitive host, in which sexual reproduction takes place, might be expected to restrict their evolutionary divergence (Maynard Smith, 1966). The phylogenetic proximity of F. hepatica and F. gigantica has been recently underscored by the finding that the transcriptomes of $F$. gigantica and $F$. hepatica showed homology (Blastx, $E<1 \mathrm{E}^{-05}$ ) for almost $90 \%$ of the protein sequences examined (Young et al., 2011). Where intermediate hosts are present which can support both F. hepatica and F. gigantica, such as in eastern Asia, hybrids between the species have been reported (reviewed in Mas Coma et al., 2009). Such hybrids may be diploid, mixoploid or triploid and are generally aspermic. Some diploid isolates of F. hepatica may also be aspermic, suggesting that parthenogenic reproduction is relatively common in wild populations of fasciolids (Itagaki et al., 2009; Hanna et al., 2008). In studies where both mitochondrial and nuclear genes have been examined (Agatsuma et al., 2000; Itagaki et al., 2005a, b) it has been shown that the mitochondrial genomes of hybrid flukes from Japan and Korea may be of either F. hepatica or F. gigantica in origin as may their nuclear genes. In contrast, with flukes from Vietnam the mitochondrial sequences were exclusively of $F$. gigantica origin (Le et al., 2008). These differences indicate that hybridisation events are relatively common and have occurred independently on several occasions. Recently infra-populations of fasciolid flukes from Egypt and Iran have also been shown to contain individuals bearing nuclear genes derived from both F. hepatica and F. gigantica (Amer et al., 2011; Amor et al., 2011). Taken together, this body of work seems to indicate that where F. hepatica and F. gigantica are found in the same infra-population, hybridisation is a common 
occurrence and the ability of these hybrids to reproduce parthenogenically allows the establishment of essentially clonal hybrid field populations. The aim of much of this work has been to determine whether flukes from a particular region should be regarded as F. hepatica or F. gigantica as this distinction is of importance with regard to epidemiology (Mas-Coma et al., 2005). However, in view of the range of reproductive strategies which may be operating in fasciolid populations (Fletcher et al., 2004) it is questionable how well the species concept (as defined by Mayr, 1963) can be applied to these trematodes (Kunz, 2002; de Meeûs et al., 2003). Moving from theoretical to practical matters, control and therapy of fasciolosis in the foreseeable future is likely to remain dependent on anthelmintic chemotherapy (Fairweather, 2011) or the development of a vaccine (McManus and Dalton, 2006). For these strategies to be applied successfully in the different regions where fasciolosis is a problem it is important that we have as full an understanding as possible of the variability inherent in the target liver fluke populations.

Working with mitochondrial genomic material it has been shown that $F$. hepatica populations can exhibit a high level of "intraspecific" diversity/divergence within a relatively confined geographic region (Walker et al., 2007, 2011) and that $F$. hepatica-like flukes can be found in geographical proximity to F. gigantica in highland regions of eastern Africa where the local climatic conditions favour the establishment of its preferred intermediate host, Lymnaea (Galba) truncatula (Walker et al., 2008). The most parsimonious explanation for the origin of the highland eastern African flukes is that they have been introduced relatively recently with livestock of European origin. In order to determine more precisely the relationship of these flukes to other populations of F. hepatica and F. gigantica a portion of the of their lsrRNA (nLSU/28S rRNA) (Teofanova et al., 2011) was sequenced as an 
113 example of nuclear genomic material. This has been supplemented with sequences

114 from the highly informative mitochondrial genome region coding for Cox III, tRNA-

115 His and Cob (Walker et al., 2007) to determine mitochondrial lineages. Phylogenetic

116 tools have been applied to determine whether the fasciolids in our African samples

117 and in additional material from Europe and India could be characterised as either F.

118 hepatica or F. gigantica. We believe that this is the first time that such an extensive

119 dataset, in terms of geographic origin and number of samples $(n=200)$ has been

120 analysed in this manner. Both nuclear and mitochondrial datasets indicate that the

121 division of these fasciolids into two species is simplistic and may conceal the

122 occurrence of cryptic speciation.

\section{2. Materials and methods}

\subsection{Sources of fasciolid specimens}

All samples of adult fasciolid worms were collected from abattoirs and thus

130 Ghaysu (Egypt). Details of the Tanzanian collection sites have been presented

131 elsewhere (Walker et al., 2008). Indian samples were collected from Chennai

132 (southern India) and Aligarh (northern India). Flukes of (ultimately) European origin

133 were obtained from Ireland, The Netherlands, Greece and Australia. All flukes from

134 regions below 2,500 metres in Tanzania originated from cattle: three from Iringa,

135 three from Mbeya and one from Njombe. Highland (>2,500 m) Tanzanian flukes

136 were from a single infrapopulation found in a cow from the Kitulo region. All adult

137 flukes collected from India originated from water buffalo; one from Aligarh and one 
138 from Chennai. Only one infrapopulation was sampled from Egypt, which was

139 harboured in a donkey. Flukes from Ireland were obtained from a cow whereas fluke

140 samples from The Netherlands (one), Greece (two) and Australia (one) were from

141 sheep. Where sufficient numbers of flukes were available, up to 24 flukes from each

142 infra-population were used for subsequent molecular analysis. All material was

143 initially washed in distilled water prior to storage in $99 \%$ molecular grade ethanol.

144

145

146

147

148

149

150

151

152

153

154

155

156

157

162

\subsection{Identification of fasciolid specimens}

Flukes were initially classified on a morphological basis and their body length and width recorded (Walker et al., 2008). Flukes morphologically similar to F.

gigantica had a mean body width to body length ratio of 2.85 whereas those

morphologically similar to F. hepatica had a ratio of 1.59. Fasciolid species were later identified according to the PCR-Restriction Fragment Length Polymorphism protocol of Marcilla et al. (2002), based on the 28S rDNA gene.

\subsection{DNA extraction from adult liver flukes}

Approximately $25 \mathrm{~mm}^{2}$ of fluke tissue were placed into $500 \mu \mathrm{l}$ of $10 \%$ Chelex ${ }^{\circledR}$ (Fluka) solution incorporating $10 \mu$ l of Proteinase K (Sigma) at a concentration of $20 \mathrm{mg} / \mathrm{ml}$. This was then heated in a heat block at $55^{\circ} \mathrm{C}$ for $1 \mathrm{~h}$, followed by gentle vortexing and a further incubation at $95^{\circ} \mathrm{C}$ for $30 \mathrm{~min}$. The mixture was then gently vortexed and centrifuged at $13,000 \mathrm{~g}$ for $10 \mathrm{~s}$. A $200 \mu \mathrm{l}$ sample of the supernatant was removed and diluted 1:10 in deionised water before storage at $-20^{\circ} \mathrm{C}$.

\section{4. mtDNA analysis}


164 haplotypes, their amplification by PCR and the subsequent sequencing of the

165 amplicons have been given elsewhere (Walker et al., 2011). Briefly, this comprised

$1661,400 \mathrm{bp}$ of contiguous mtDNA enclosing the regions coding for cytochrome $\mathrm{c}$

167 oxidase subunit III (cox III), transfer RNA histidine (tRNA-His) and cytochome b

168 (cob). Two primer sets were used to generate overlapping fragments in PCR: Primer

169 set 1: Fhmt1.1F 5'-gcttgtgggttttcttaggg-3', Fhmt1.1R 5'-caaccaaacctcaacaacct-3';

170 Primer set 2: Fhmt1.2F 5'-tgtggtgtcggagagttctg-3' and, Fhmt1.2R 5'-

171 taaccataggatccgectga-3. Fragment 1 consisted of nucleotides 77 to 881 of the

172 complete F. hepatica mitochondrial sequence (Le et al., 2001), whilst fragment two

173 ran from nucleotides 681 to 1,480 . As the second primer set failed to amplify the

174 morphologically F. gigantica-like flukes, an additional primer set was designed, Fgmt

175 1.2F 5'-ggtgtcggagagttctgttg-3' and Fgmt1.2R 5'-accaaatcaggaaacaccaa-3'. PCR

176 products were purified as detailed elsewhere (Walker et al., 2011) and sequenced

177 commercially by Macrogen (Korea). The sequences comprising the non-African $F$.

178 hepatica dataset have been published previously (Walker et al., 2011; Teofanova et

179 al., 2011) and accession numbers may be obtained from those sources. The Indian

180 and African datasets have the following accession numbers: Aligarh, $\underline{\text { JX236561- }}$

181 JX236579; Chennai, $\underline{\mathbf{J X 2 3 6 5 8 0 - J X 2 3 6 5 9 0}}$; Iringa, JX236591-JX236639; Kitulo,

182 JX236643-JX236655; Mbeya, $\underline{\mathbf{J X 2 3 6 5 0 8 - J X 2 3 6 5 6 0}}$; Njombe, JX236640-

183 JX236642.

184

185

2.5. rDNA analysis

186

Primers 28SF 5' - agctgattaccegctgaact-3' and 28SR 5'-

187 ctgagaaagtgcactgacaag-3'were used for amplification of the region from $15 \mathrm{bp}$ to 632 
188 bp (618 bp in length), with GenBank accession number $\underline{\mathbf{A J 4 4 0 7 8 8}}$ (F. hepatica partial

189 28SrRNA gene, Bolivia: Northern altiplano) (Marcilla et al., 2002) using the PCR

190 conditions described by Teofanova et al. (2011). PCR products were purified and

191 sequenced as above.

192

193

\subsection{Data Analysis}

194 Raw sequencing data (both directions in each case) were initially assembled

195 using ChromasPro (Technelysium Pty. Ltd, Australia) software and subsequent

196 alignments were carried out in BioEdit (Hall, 1999). All sequencing variants were

197 double checked on chromatogram traces before the derived sequences were allowed to

198 progress for further analysis. DNAsp (Rozas et al., 2003) was used to calculate the

199 haplotype diversity, average number of nucleotide differences between sequences and

200 nucleotide diversity (Nei, 1987) for each sample. To investigate the phylogenetic

201 relationships and relative frequency among the resulting mtDNA haplotypes, taking

202 into consideration the geographic origin of the samples, a haplotype network was

203 constructed using the median-joining method (Bandelt et al., 1999), implemented in

204 Network 4.5.0.2 (fluxus-engineering.com, Fluxus Technology Ltd., UK, 2004). A

205 Bayesian phylogenetic analysis on the resulting mtDNA haplotypes was also

206 performed using MrBayes version 3.2 (Ronquist and Huelsenbeck, 2003) using the

207 GTR + G model of DNA substitution, estimated using MODELTEST v3.0, Akaike

208 Information Criterion (Posada and Crandall, 1998). Four replicates of the Markov

209 chain Monte Carlo (MCMC) search were run with four chains of 10,000,000

210 generations each. Trees were sampled every 1,000 generations following a burn-in of

21125,000 generations for each replicate. A Fasciola jacksoni sequence was used as an

212 outgroup for the analysis. In an attempt to provide a quantitative assessment of 
213 whether the differentiation between the mtDNA groups/clades identified from the

214 phylogenetic analyses was sufficient to confirm the existence of distinct species, the

215 data were analysed according to the " $4 \mathrm{x}$ rule" species criterion suggested by Birky et

216 al. (2010). This approach is designed to identify clusters that are separated by $t \geq 4 N_{e}$

217 generations (where $t$ is the time to most recent common ancestor and $N_{e}$ is the

218 effective population size), which is equivalent to the upper $95 \%$ confidence limit of

219 the coalescent time and the depth of separation formed by random drift. For two such

220 clades the ratio of divergence between individuals from each clade $(K)$ and $\theta$, the

221 Watterson estimator of population mutation rate, is given by the equation $K / \theta=$

$2222 t \mu / 2 N_{e} \mu \geq 8 N_{e} \mu / 2 N_{e} \mu=4$. Although we do not have an accurate measure of $N_{e}$ or

223 the mutation rate $(\mu)$, these can be assumed to be the same for each clade and can thus

224 be eliminated from the equation. Speciation may therefore be considered to have

225 occurred (by this criterion) when the mean sequence divergence $(K)$ between

226 individuals in two candidate clades is greater than $4 * \theta$, where $\theta[\theta=\pi /(1-4 \pi / 3)]$ is

227 derived from the mean sequence difference between individuals within a clade, $\pi$; this 228 parameter can be calculated from our sequence dataset.

230 3. Results

231

232 3.1. Differentiation according to morphology and $28 S$ rDNA

233 Morphological criteria assigned all the Indian flukes to F. gigantica. All of

234 the European flukes were classified as F. hepatica as were the African flukes from

235 Kitulo (Tanzania) and Egypt. All other African flukes were of the F. gigantica

236 phenotype. 
The analysis of the 618 bp fragment from $28 \mathrm{~S}$ rDNA is shown in Table 1.

238 There were four positions at which nucleotide variation was observed. These

239 occurred at positions 105, 130, 283 and 547. This variation was not in complete

240 accordance with the morphological determination of species. At position 105, a

241 substantial minority of the European and Egyptian flukes carried a G, as did all of the

242 Indian and most of the Tanzanian flukes. At locus 130, all but one of the European,

243 Australian and Egyptian flukes carried an A as did the flukes from Kitulo whilst the

244 Indian and other African flukes had a $\mathrm{G}$ at this position. However, one European and

245 one Tanzanian fluke were heterogeneous at this site. Position 283 distinguished

246 between African "F. gigantica" and Indian "F. gigantica", the former having a G as

247 opposed to the A seen in the other populations. Again, two heterogeneous flukes

248 were observed. Position 547 gave the best agreement with morphological

249 classification with the $F$. hepatica-like flukes bearing a $C$ at this position and the $F$.

250 gigantica-like samples a T, although again two aberrant samples were noted. The

251 heterogeneity seen at positions 130, 283 and 547 in the European and Iringa

252 populations was due in each case to an individual fluke showing two alleles at each 253 position.

255 3.2. Mitochondrial haplotype analysis

256 The fluke samples which provided mitochondrial sequence data are shown in

257 Table 2 together with statistical data relating to the sequences. In general, this region

258 of the mitochondrial genome was extremely variable with the number of haplotypes

259 observed being approximately one-third of the sample size. The population from the

260 highland region of Tanzania (Kitulo) was the most diverse with a very high average

261 number of nucleotide differences between paired sequences and the highest levels of 
262

263

nucleotide diversity; this was in contrast to the population from the lowland regions of Tanzania which was the least diverse.

A Bayesian tree constructed using the sequences of the unique mitochondrial haplotypes and rooted using the homologous region from F. jacksonii is shown in Fig. 1. The F. gigantica- and F. hepatica- like flukes were clearly separated into two deeply rooted clades. Within the "gigantica" clade there was a further division between the F. gigantica-like flukes from Africa and those from India. A division of comparable or greater depth existed between a subset of the highland (Kitulo) flukes and the rest of the F. hepatica-like population. There was strong posterior probability support $(\mathrm{P}=1)$ for each of these groupings. Within the $F$. gigantica African clade and the "European" F. hepatica clade there was little geographical structuring.

$$
\text { In order to substantiate these findings through the use of an alternative }
$$
analytical tool, a Median Joining network was constructed using the mitochondrial dataset (Fig. 2). This employs a different algorithm and allows for the simultaneous existence of ancestral and derived haplotypes in the network - this is not possible using trees. This analysis indicated that there were four clearly separated clades based on geographic origin but with a subset of the highland Tanzanian flukes being more closely associated with the European population than others from the Kitulo region. The two F. hepatica-like clades were separated by almost three times the number of nucleotide substitutions separating the Indian and African F. gigantica-like clades.

\subsection{Application of the $4 x$ rule for speciation}

The population statistics used to test for speciation in accordance with the $4 \mathrm{x}$ rule model are presented in Table 3. With the exclusion of the subset of highland 
286 Tanzanian flukes, which clustered with the European F. hepatica population, it can be 287 seen that each clade meets this criterion for speciation.

\section{Discussion}

This study was prompted by our interest in the morphologically F. hepatica-

291 like liver flukes found in association with Lymnaea truncatula, the favoured

292 intermediate host for F. hepatica, in areas of the Tanzanian highlands above $2,500 \mathrm{~m}$

293 (Walker et al., 2008). rDNA genes have been extensively employed as

294 representatives of the nuclear genome in studies of speciation in digeneans (Lotfy et

295 al., 2008) and the partial 28S subunit has been reported to be suitable for use in

296 distinguishing between F. hepatica and F. gigantica (Marcilla et al., 2002). Liver

297 flukes from eastern Europe are known to contain two 28S genotypes (Tefanova et al.,

298 2011) and the relative proportions of these has been shown to correlate with

299 environmental temperature when used in ecological niche modeling (Kantzoura et al.,

300 2011). Analysis of the partial $28 \mathrm{~S}$ sequences (Table 1) at the 130 position gave good

301 discrimination between morphologically hepatica-like and gigantica-like flukes with

302 hepatica-like flukes carrying the 130A genotype whilst the gigantica-like flukes

303 carried a 130G transition. Position 283 was of interest in that it distinguished between

304 the gigantica-like flukes of African origin and those from India, and position 547 was

305 comparable to position 130 in its ability to discriminate between hepatica-like flukes

306 and gigantica-like flukes. Taken in toto the results from the analysis of the variable

307 positions in $28 \mathrm{~S}$ rDNA indicate that the nuclear genome of the highland Tanzanian

308 flukes is related to that of the subset of F. hepatica of European origin which has been

309 associated with tolerance of high temperatures (Kantzoura et al., 2011) but they also

310 reveal that F. gigantica from Africa and India may differ in their nuclear genomes. 
311 This may be of importance as to date most research on F. gigantica has been

312 undertaken with material from Asia with its applicability to African F. gigantica

313 being assumed.

314 As expected, the mitochondrial genome region analysed was highly

315 informative with regard to intra-population differences. The statistics presented in

316 Table 2 indicate that the F. hepatica-like flukes from the Tanzanian highland region

317 were by far the most diverse genetically. This was in contrast to the F. gigantica-like

318 Tanzanian flukes which provided the least genetically diverse group. It has been

319 proposed that F. gigantica originated in the east African lowlands and spread to Asia

320 following the domestication of bovids (Mas Coma et al., 2009). In general, more

321 recently colonised areas have lower genetic variability (Hewitt, 2000). The ranking

322 order of liver fluke genetic diversity seen in Table 2 (European $>$ Indian $>$ African

323 lowland) might be taken to imply that African populations of F. gigantica are less

324 ancient than those of India but our results may be influenced more by the size of the

325 geographic region from which the samples were collected than their relative age. A

326 confounding factor may be the frequency with which population bottle-necks may

327 have occurred - for example, Europe, southern Africa and India differ greatly in their

328 history of glacial cycles. An explanation of the high level of genetic diversity seen in

329 the highland Tanzanian flukes was provided by the analyses presented in Figs. 1 and 2

330 which show that this population is made up of two distinct clades. One of these was

33120 nucleotide differences removed from the European F. hepatica clade whereas the

332 other was over 70 substitutions distant (Fig. 2).

333 The mitochondrial data supported the distinction seen with the nuclear 28S

334 rDNA gene in the African and Indian F. gigantica-like flukes in that these populations

335 were separated into two well supported clades. There was a deep separation between 
336 the morphologically F. hepatica-like and the F. gigantica-like flukes, with the former

337 clade showing further sub-division into the flukes of ultimately European origin

338 (Netherlands/Ireland/Greece/Australia/Egypt (Mas Coma et al., 2009)) compared with

339 those from the Tanzanian highlands. Four of the five haplotypes present in the

340 Tanzanian highland population formed a clade separated from the European flukes by

341 at least 71 nucleotide substitutions (Fig. 2). Relating such data to a molecular clock is

342 hazardous for reasons discussed in detail elsewhere (Walker et al., 2011), but

343 assuming a rate of change for F. hepatica comparable with that reported for

344 Schistosoma mansoni (Morgan et al., 2005) of 4\% per million years, these 71

345 substitutions would be equivalent to approximately 1 million years, ruling out the

346 possibility that they could have been introduced as parasites present in domesticated

347 herbivores. The remaining haplotype present in the highland Tanzanian fluke

348 population was represented by four flukes and was within the range of nucleotide

349 diversity seen in European F. hepatica. As such it may have been introduced together

350 with the cattle and sheep known to have been brought into this area in recent times

351 (Walker et al., 2008).

352 Little evidence was seen that would indicate the presence of hybrid flukes in

353 our populations. In all but two individuals the mitochondrial haplotype was

354 consistent with the nuclear 28S rDNA genotype. These aberrant flukes from the

355 European and Iringa populations were heterozygous at 28S rDNA positions 130, 283

356 and 547 which could indicate hybridization between an F. hepatica- like fluke and an

357 African F. gigantica- like fluke in previous generations. Whilst this is possible for the

358 Tanzanian fluke, it seems less likely in the case of the European fluke as it was from

359 an infrapopulation present in a Dutch sheep and would thus be distant from known

360 sources of F. gigantica. 
The results presented in this study show that the four geographical populations

362 of Fasciola spp. can be assigned to four clades which are well separated genetically.

363 Application of the $4 \mathrm{x}$ rule for speciation (Table 3) suggested that these clades are

364 sufficiently divergent to be regarded as species under the definition forming the basis

365 of this model (Birky et al., 2010). However, it has been proposed that in the absence

366 of an internationally agreed definition of a species that is applicable to parasites we

367 should refrain from naming new species "just on the basis of a certain number of base

368 exchanges within their ribosomal DNA sequence" (Kunz, 2002). Fasciola gigantica

369 and F. hepatica are well established as separate species and can be distinguished by a

370 number of characteristics - morphologically (Periago et al., 2006), by their

371 preferences for intermediate hosts (Radix natalensis does not appear to be capable of

372 supporting the growth of F. hepatica (Boray, 1985)), the minimal temperature

373 required for egg hatching (Grigoryan, 1958; Ross and McKay, 1929) and by the

374 ability of F. hepatica to evade immune responses in the Indonesian Thin-Tailed sheep

375 (Roberts et al., 1997). If the Tanzanian F. hepatica-like flukes and African F.

376 gigantica-like flukes are separated from these populations due to the process of

377 speciation we might expect to discover differences of comparable importance to their

378 biology on closer examination (Nicolalde-Morejon et al., 2009; Nadler and Perez-

379 Ponce de Leon, 2011). Indeed, prior to 1965 (when it was synonymised with F.

380 gigantica (Kendall, 1965)) liver flukes from India and elsewhere in south Asia were

381 classified as Fasciola indica (Varma, 1953). This distinction was made on

382 morphological grounds, with their spines and eggs in particular showing distinct

383 differences from both European F. hepatica and African F. gigantica. With further

384 research on the four clades identified in this study it may become apparent that there 
385 are distinct differences in their physiology, behaviour or morphology. If this is the

386 case then it may be pragmatic at that stage to designate them as separate species.

387 In conclusion, it has been shown that four populations of Fasciola spp. from

388 hosts located in geographically and climatically different environments form

389 divergent lineages. It is to be presumed that the selective pressures which have

390 brought about this situation may also have led to the evolution of other adaptive

391 differences between the lineages. These may be expressed as biological

392 characteristics such as host preference, tolerance to high or low temperatures,

393 sensitivity to anthelmintics and immunoevasive mechanisms, all of which could be of

394 importance to the future spread and control of fasciolosis.

395

\section{Acknowledgements}

397 We would like to thank Professor P Ramasamy (University of Alagappa,

398 India) and Professor SMA Abidi (Aligarh Muslim University, India) for their

399 assistance in the collection of flukes. This study was supported in part by the DeLiver

400 consortium of the European Union VII Framework Programme (FOOD-CT-200X-

401 023025) and a British Society for Parasitology Ann Bishop Award (2006) to SMW.

402 The funders had no role in study design, data collection and analysis, decision to

403 publish or preparation of the manuscript.

404

405 References

406

407 Agatsuma, T., Arakawa, Y., Iwagami, M., Honzako, Y., Cahyaningshi, U., Kang,

408 S.Y., Hong, S.J., 2000. Molecular evidence of natural hybridization

409 between Fasciola hepatica and F. gigantica. Parasitol. Int. 49, 231-238. 
411 Amer S., Dar Y. Identification of Fasciola species isolated from Egypt based on sequence analysis of genomic (ITS1 and ITS2) and mitochondrial (NDI and COI) gene markers. Parasitol Int. 60, 5-12.

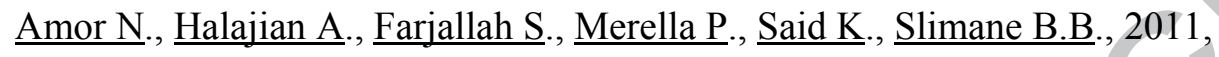
Molecular characterization of Fasciola spp. from the endemic area of northern Iran based on nuclear ribosomal DNA sequences. Exp Parasitol.

Birky C.W. Jr, Adams J., Gemmel M., Perry J., 2010, Using population genetic $\underline{\text { theory and DNA sequences for species detection and identification in }}$ asexual organisms. PLoS One. 5(5) e10609.

Fairweather, I. 2011, Reducing the future threat from (liver) fluke: realistic

Boray, J.C., 1985. Flukes of domestic animals. In: Gaafar, Howard, Marsh (Eds.), 
435

436

437

438

439

440

441

442

443

444

445

446

447

448

449

450

451

452

453

454

455

456

457

458

459

Grigoryan G.A., 1958, Experimental study of Fasciola gigantica infestation in sheep. Trudy Armyansk Inst Zhivotnovod i Veterinariya 3, 155-168.

Hall T.A., 1999, BioEdit: a user-friendly biological sequence alignment editor and analysis program for Windows 95/98/NT. Nucl Acids Symp Ser 95-98.

Hanna R.E., Edgar H., Moffett D., McConnell S., Fairweather I., Brennan G.P., Trudgett A., Hoey E.M., Cromie L., Taylor S.M., Daniel R., 2008, Fasciola hepatica: Histology of the testis in egg-producing adults of several laboratory-maintained isolates of flukes grown to maturity in cattle and sheep and in flukes from naturally infected hosts. Vet Parasitol. 157, 22234.

Hewitt G., 2000, The genetic legacy of the Quaternary ice ages. Nature. 405, 90713.

Itagaki, T., Kikawa, M., Sakaguchi, K., Shimo, J., Terasaki, K., Shibahara, T., Fukuda, K., 2005a. Genetic characterization of parthenogenetic Fasciola sp. in Japan on the basis of the sequences of ribosomal and mitochondrial DNA. Parasitology 131, 679-685.

Itagaki, T., Kikawa, M., Terasaki, K., Shibahara, T., Fukuda, K., 2005b. Molecular characterization of parthenogenic Fasciola sp. in Korea on the basis of DNA sequence of ribosomal ITS1 and mitochondrial NDI gene. J. Vet. Med. Sci. 67, 1115-1118. 
461 Itagaki T., Sakaguchi K., Terasaki K., Sasaki O., Yoshihara S., Dung T.V.., 2009.

462 Occurrence of spermic diploid and aspermic triploid forms of Fasciola in

463 Vietnam and their molecular characterization based on nuclear and

464 mitochondrial DNA. Parasitol. Int. 58, 81-85.

465

466 Kantzoura V., Kouam M.K., Feidas H., Teofanova D., Theodoropoulos G., 2011,

467 Geographic distribution modelling for ruminant liver flukes (Fasciola

468 hepatica) in south-eastern Europe. Int. J. Parasitol. 41 747-53.

469

470 Kendall, S.B., 1965. Relationships between the species of Fasciola and the

471 molluscan hosts. Adv. Parasitol. 3, 59-98.

472

473 Kunz, W., 2002. When is a parasite species a species? Trends Parasitol. 18, 121-

$474 \quad 124$.

475

476 Le, T.H., De, N.V., Agatsuma, T., Nguyen, T.G.T., Nguyen, Q.D., McManus,

477 D.P., Blair, D., 2008. Human fascioliasis and the presence of

478 hybrid/introgressed forms of Fasciola hepatica and Fasciola gigantica in

479 Vietnam. Int. J. Parasitol. 38, 725-730.

480

481 Lotfy W.M., Brant S.V., DeJong R.J., Le T.H., Demiaszkiewicz A., Rajapakse

482

483

484
R.P., Perera V.B., Laursen J.R., Loker E.S., 2008. Evolutionary origins, diversification, and biogeography of liver flukes (Digenea, Fasciolidae). Am J Trop Med Hyg. 79, 248-55 
486 Marcilla A., Bargues M.D., Mas-Coma S., 2002. A PCR-RFLP assay for the 487 distinction between Fasciola hepatica and Fasciola gigantica. Mol. Cell. $488 \quad$ Probes 16, 327-33.

489

490 Mas-Coma S., Bargues M.D., Valero M.A., 2005. Fascioliasis and other plant-

$491 \quad$ borne trematode zoonoses. Int. J. Parasitol. 35, 1255-78.

492

493 Mas-Coma, S., Valero, M.A., Bargues, M.D., 2009. Fasciola, lymnaeids and

494 human fascioliasis, with a global overview on disease transmission,

495 epidemiology, evolutionary genetics, molecular epidemiology and control.

496 Adv. Parasitol. 69, 41-146.

497

498 Maynard Smith, J., 1966. Sympatric speciation. Am. Nat. 100, 637 -650.

499

500 McManus D.P. and Dalton J.P., 2006. Vaccines against the zoonotic trematodes

501 Schistosoma japonicum, Fasciola hepatica and Fasciola gigantica.

$502 \quad$ Parasitology 133, S43-S61.

503

504 de Meeûs, T., Durand, P., Renáud, F., 2003. Species concepts: what for? Trends

$505 \quad$ Parasitol. 19, 425-427.

506

507 Morgan J.A., Dejong R.J., Adeoye G.O., Ansa E.D., Barbosa C.S., Brémond P., 508 Cesari I.M., Charbonnel N., Corrêa L.R., Coulibaly G., D'Andrea P.S., De 509 Souza C.P., Doenhoff M.J., File S., Idris M.A., Incani R.N., Jarne P., 
$\underline{\text { Karanja D.M., Kazibwe F., Kpikpi J., Lwambo N.J., Mabaye A., }}$ Magalhães L.A., Makundi A., Moné H., Mouahid G., Muchemi G.M., Mungai B.N., Séne M., Southgate V., Tchuenté LA., Théron A., Yousif F., Zanotti-Magalhães E.M., Mkoji G.M., Loker E.S., 2005. Origin and diversification of the human parasite Schistosoma mansoni. Mol. Ecol. 14, 3889-902.

Nadler S.A., Ponce de León G., 2011. Integrating molecular and morphological approaches for characterizing parasite cryptic species: implications for parasitology. Parasitology. 138, 1688-709.

Nicolalde-Morejón, F., Vergara-Silva, F., González-Astorga, J., Vovides, A.P., Espinosa de los Monteros, A., 2009. Reciprocal illumination of morphological characters upon a molecular hypothesis supports the proposal of a new species of cycad from Mexico. Systemat. Biodiver. 7, 73-79.

Nei, M., 1987. Molecular evolutionary genetics. (chapter 10) Columbia University Press, New York.

(

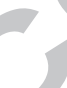
comparison of allopatric populations of Fasciola hepatica and Fasciola gigantica from European and African bovines using a computer image analysis system (CIAS). Parasitol. Res. 99, 368-378. 
535 Posada, D., Crandall, K.A. 1998. MODELTEST: testing the model of DNA 536 substitution. Bioinformatics. 14, 817-818.

537

538 Roberts, J.A., Estuningsih, E., Widjayanti, S., Wiedosari, E., Partoutomo, S., 539 Spithill, T.W., 1997. Resistance of Indonesian thin tail sheep against $540 \quad$ Fasciola gigantica and F. hepatica. Vet. Parasitol. 68, 69-78.

541

542

543

544

545

546

548

549

550

551

552

553

554

556

557

Ronquist, F., Huelsenbeck, J.P. 2003. MrBayes 3: Bayesian phylogenetic inference under mixed models. Bioinformatics. 19, 1572-1574.

Ross I.C., McKay, A.C., 1929. The bionomics of Fasciola hepatica in New South Wales and of the intermediate host Limnaea brazieri. Bulletin of the Council of Scientific and Industrial Research Australia. No 43.

Rozas J., Sánchez-DeBarrio, J.C., Messeguer, X., Rozas, R. 2003. DnaSP. DNA polymorphism analyses by the coalescent and other methods. Bioinformatics. 19, 2496-2497.

Teofanova, D., Kantzoura, V., Walker, S., Radoslavov, G., Hristov, P., Theodoropoulos, G., I. Bankov, I., Trudgett, A., 2010. Genetic diversity of liver flukes (Fasciola hepatica) from Eastern Europe. Infect. Genet. Evol. 11. 109-15. 
558 Torgerson, P., Claxton, J., 1999. Epidemiology and control. In: Dalton (Ed.),

559 Fasciolosis, CAB International Publishing, Wallingford, United Kingdom,

$560 \quad$ pp. 113-149.

561

562 Varma, A.K., 1953. On Fasciola indica n. sp. with some observations on F.

563 hepatica and F. gigantica. J. Helminthol. 27, 185-198.

564

565 Walker, S.M., Prodöhl, P.A., Fletcher, H.L., Hanna, R.E.B., Kantzoura, V., Hoey

566 E.M., and Trudgett, A., 2007. Evidence for multiple mitochondrial lineages

567 of Fasciola hepatica (liver fluke) within infrapopulations from cattle and

568 sheep. Parasitol. Res. 101, 117-125.

569

570 Walker, S.M., Makundi,A.E., Namuba, F.V., Kassuku A.A, Keyyu, J., Hoey, E.,

571 Prödohl, P., Stothard, JR and Trudgett, A., 2008. The distribution of

$572 \quad$ Fasciola hepatica and Fasciola gigantica within southern Tanzania -

$573 \quad$ constraints associated with the intermediate host. Parasitology 135, 495-

574503.

575

576 Walker, S.M, Johnston' C., Hoey E.M., Fairweather' I., Borgsteede, F., Gaasenbeek,

577 C., Prodöhl' P.A., A. Trudgett' A., 2011. Population dynamics of the liver

578 fluke, Fasciola hepatica: the effect of time and spatial separation on the

579 genetic diversity of fluke populations in the Netherlands. Parasitology 138,

$580 \quad 215-23$.

581 
582 Young N.D., Jex A.R., Cantacessi C., Hall R.S., Campbell B.E., Spithill T.W.,

583 Tangkawattana S., Tangkawattana P., Laha T., Gasser R.B., 2011. $\underline{\text { A }}$

584 portrait of the transcriptome of the neglected trematode, Fasciola

585 gigantica--biological and biotechnological implications. PLoS Negl Trop

$586 \quad$ Dis. 5(2):e1004.

587 
590 Fig. 1. Bayesian phylogenetic tree of liver fluke (Fasciola gigantica and Fasciola 591 hepatica) mtDNA haplotypes (branch lengths are proportional to the number of 592 inferred changes). Different colours/shades represent the geographic origin of 593 clades/groups identified in this study. Nodal values represent posterior probability 594 support for particular groups. Only values higher than 0.52 are shown. Taxon name 595 codes: Fh, Fasciola hepatica; Fg, Fasciola gigantica; Du. Netherlands; In, India; S, 596 south; N, north; Tz, - Tanzania; I, Iringa; M, Mbeya; Eur, Europe (Ireland/Greece);

597 Ob, Australia. Fasciola jacksonii served as an outgroup.

598

599 Fig. 2. Median Joining Network for liver fluke (Fasciola gigantica and Fasciola 600 hepatica) mtDNA haplotypes. Different colours/shades represent the geographic 601 origins of clades/groups identified in this study. Sizes of nodes are proportional to 602 frequency of the haplotype within the dataset and distance between nodes within a 603 group is approximately proportional to the number of nucleotide changes between 604 haplotypes. For clarity of presentation the minimal number of nucleotide 605 substitutions between groups is indicated rather than displayed to scale. 
606

607

608

609

610

\section{Highlights}

Fasciola hepatica and Fasciola gigantica from Africa, Europe and India have been compared

The analyses support the existence of four distinct clades

Cryptic speciation may have occurred, masking significant biological differences 
F. gigantica (India)

F. gigantica (Africa)

F hepatica (Europe)

F. hepatica (Africa)

1.0

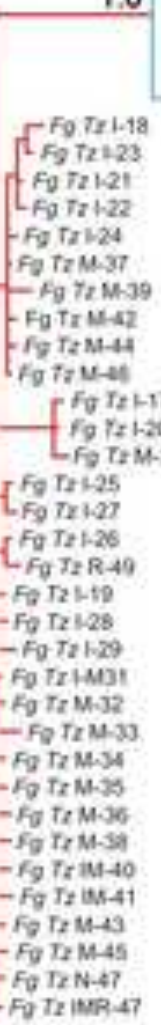

$-F_{2}$ is N.7
- Folin $\mathrm{N} B$

fgin $N-10$

$-F g$ in $\$ 12$.

- Fo in 5.45

F 72123

Fo $72+22$

$\log _{\tan 24}$

Folz

$x_{2} M-42$

uat

$y=1+20$

gram-so

Tring

Fot $T=26$

fotem.

G 7 rivito

Thin-es

Fo 72 IMR 47

Finfort

Fheors

0.96 Ph Eun

I Fin Eunto

0.94 L $F_{n=0.12}$

Fh Eving

0.87 Fon 0011

Fli Eien

0.92 - An Eun

ए.92.

ThDu-13

1.0 


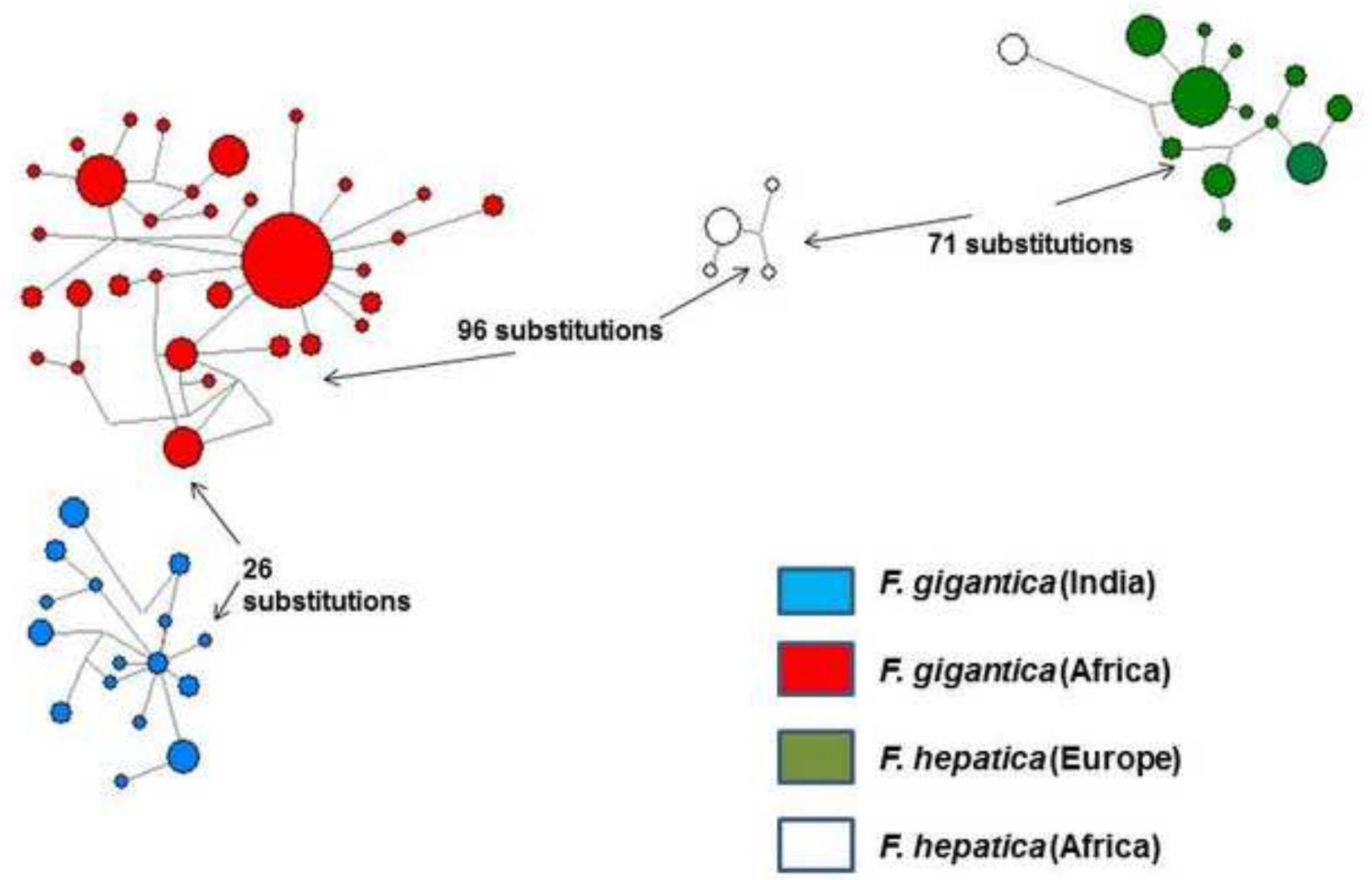

F. hepatica(Africa) 
611

612

\section{Table 1}

614 28S rDNA diversity for fasciolids from different geographic regions.

615

616

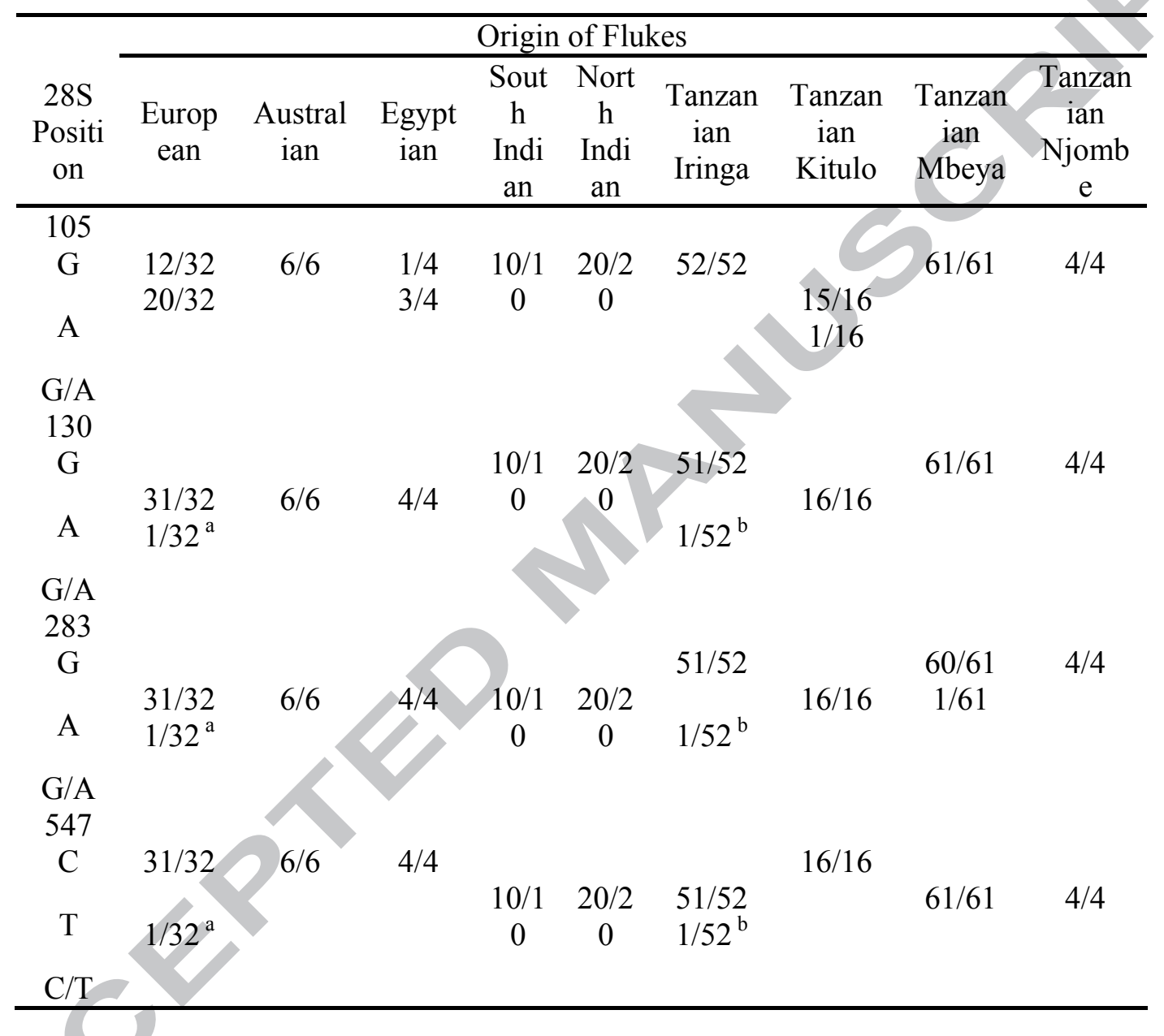

617

618 Numbers indicate the number of flukes within a set (numerator) and the number from 619 the respective geographic region sampled (denominator).

620 a Due to the same Dutch fluke.

$621{ }^{\mathrm{b}}$ Due to the same Iringa fluke.

622 


\section{Table 2}

Population and mitochondrial genome genetic statistics for fasciolids from different geographic regions.

627

\begin{tabular}{lllllll}
\hline & & No. & & Ave no. & & \\
No. & polymorphic & No. & nucleotide & Nucleotide & Standard & Haplotype \\
diversity & differences & diversity & deviation & dives \\
\hline
\end{tabular}


631 Table 3

632 Variables associated with the populations used to test compliance with the " $4 \mathrm{x}$ rule"

633 for speciation.

634

635

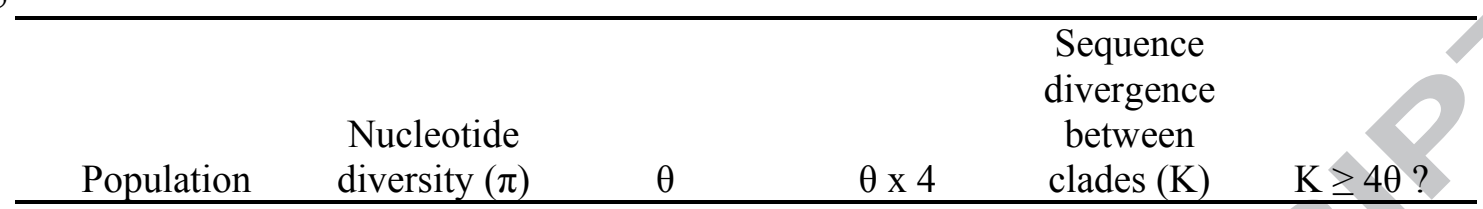

All Fasciola

spp.

0.10151

European

Fasciola

hepatica

$0.00748 \quad 0.00755$

0.0302

Yes

All Fasciola

gigantica

$0.01283 \quad 0.01283$

0.05128

Yes

All F. hepatica-

like

European F.

hepatica

0.00748

0.00755

0.0302

Yes

Highland

Tanzanian $F$.

hepatica $^{\mathrm{a}}$

0.0029

0.0029

0.0116

Yes

All F. gigantica

spp.

0.03052

African $F$.

gigantica

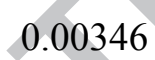

0.00346

0.01384

Yes

Indian $F$.

gigantica

0.00662

0.00663

0.02652

Yes

$637{ }^{a}$ Highland Tanzanian samples clustering with the European clade $(n=4)$ were 638 excluded.

639 


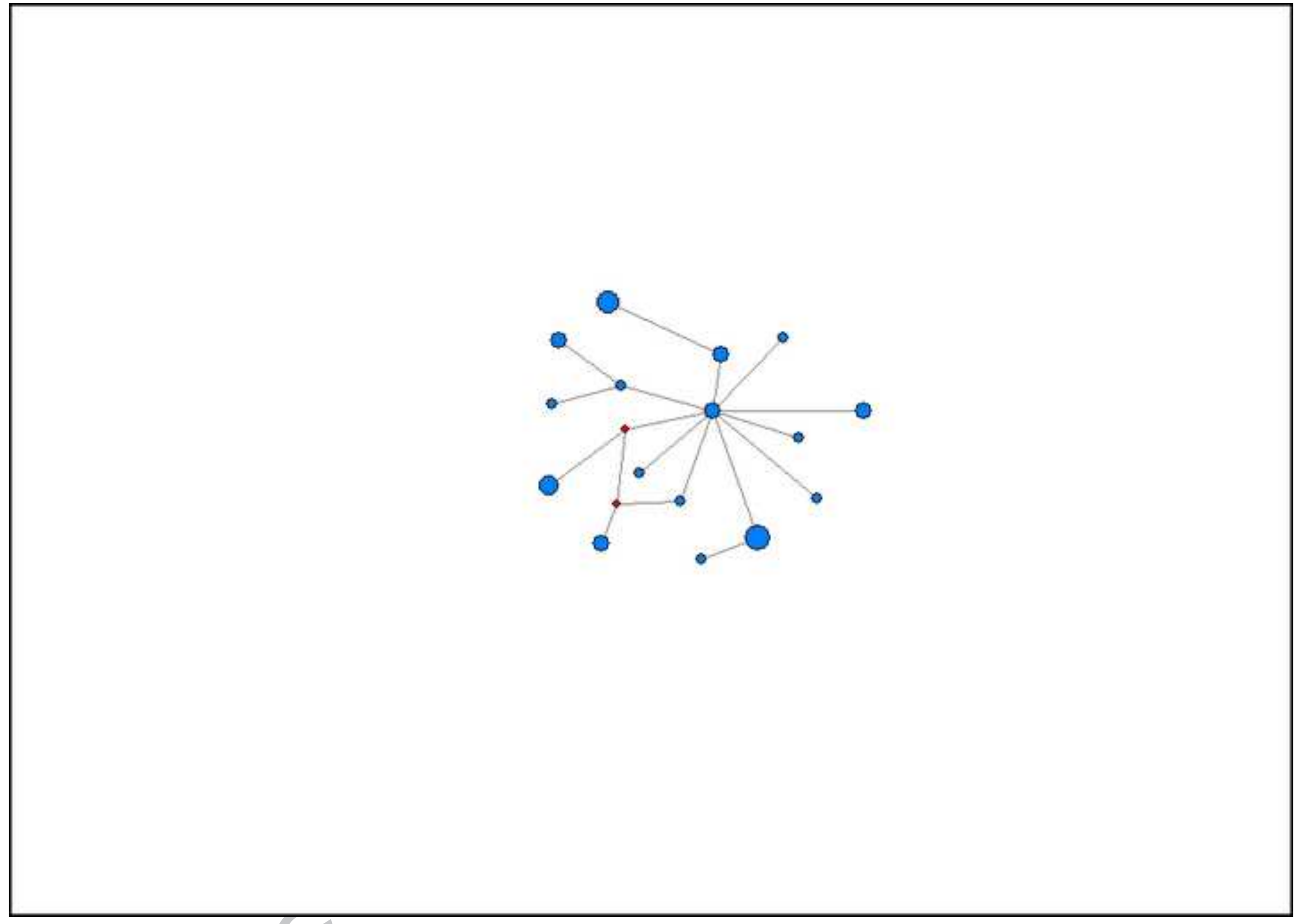

